\title{
A REPRESENTATION THEOREM AND ITS APPLICATIONS TO CHARACTERIZE BOUNDED AND COMPACT COMPOSITION OPERATORS ON BESOV SPACES ON DOMAINS IN $\mathbb{C}^{N *}$
}

\author{
HYUNGWOON $\mathrm{KOO}^{\dagger}$, SONG-YING LI ${ }^{\ddagger}$, AND SUJUAN LONG ${ }^{\S}$ \\ Dedicated to Professor Stephen Yau on the occasion of his 60th birthday
}

\begin{abstract}
We prove a representation theorem for Besov space on smooth bounded strictly pseudoconvex domain. As applications we characterize the boundedness and the compactness of composition operators on Besov spaces.
\end{abstract}

Key words. Representation theorem, Composition operator, Besov space, Strictly pseudoconvex domain.

AMS subject classifications. Primary 32A10; Secondary $47 \mathrm{~B} 33$.

1. Introduction. Given a holomorphic self-mapping $\phi$ on a domain $D$ in $\mathbb{C}^{n}$, the composition operator $C_{\phi}$ acting on function $u$ on $D$ is defined as $C_{\phi}(u)(z)=u(\phi(z))$. Theory of composition operators on holomorphic function spaces over a domain $D$ in $\mathbb{C}^{n}$ has been developed by many authors in the last four decades (for examples, see the book of Shapiro [33], the book of Cowen and MacCuler [8], the book of Zhu [39], survey papers by Li [18], Russo [31] and references therein). Major researches have been concentrated on the function theoretic characterization of bounded, compact and Schatten-von-Neumann class composition operators as well as the spectral distribution of composition operators. When $n=1$, the composition operator is always bounded on Hardy space $H^{p}(D)$ (see Li [18] for a proof and references therein). However, it is no longer true when $n>1$ and counter examples were discovered by Shapiro, and Cima, Stanton and Wogen [6], MacCluer [29], Cima and Wogen [7].

A complete function theoretic characterization of a compact composition operator on Hardy space over the unit disk was given by Shapiro [32]. For results of compact composition operators and Schatten-von-Neumann class composition operators on Bergman spaces or Hardy spaces in several complex variables can be found (for examples) from the references: [8], [9], [17], [18, 19, 20], [27], [25], [30], [31], [34] and [39].

Compact composition operators on Bloch space over classical bounded symmetric domains were characterized by Zhou and Shi [37] and compact composition operators on BMOA space over the unit disc were characterized by Bourdon, Cima and Matheson [3], Smith [35] and Wulan [36]. Characterization of compact composition operators on $B M O A\left(B_{n}\right)$ was given by Li and Long [21] and many others.

In this paper we characterize bounded and compact composition operators on holomorphic Besov space $B^{p}(D)$ over a smoothly bounded strictly pseudoconvex domain $D$ in $\mathbb{C}^{n}$. A representation theorem for $B^{p}(D)$, which we develop along the way, plays a key role in our proof and also is of independent interests itself. Such a

\footnotetext{
* Received March 4, 2013; accepted for publication October 16, 2013.

$\dagger$ Department of Mathematics, Korea University, Seoul 136-713, Korea (koohw@korea.ac.kr). H. Koo was supported by KRF of Korea (2012R1A1A2000705).

${ }^{\ddagger}$ Department of Mathematics, University of California, Irvine, CA 92697-3875, USA (sli@math. uci.edu).

$\S$ School of Mathematics and Computer Science, Fujian Normal University, Fuzhou, Fujian, China.
} 
representation for Hardy and weighted Bergman spaces on the bounded symmetric domains in $\mathbb{C}^{n}$ was given by Coifman and Rochberg in [4], and for $A_{p}$-Hardy and Bergman space as well as for BMOA and Bloch spaces over the unit ball in $\mathbb{C}^{n}$ by Luecking in [26].

To state our theorems we need to introduce function spaces and some notation. Let $K(z, w)$ be the Bergman kernel function for a domain $D$ and $K(z)=K(z, z)$. Let $d v$ be the normalized Lebesgue measure on $D$ and $d \lambda(z)=K(z) d v$ which is a biholomorphic invariant measure over $D$. For each $z \in D$, we let

$$
k_{z}(w)=K(z)^{-1} K_{z}(w), \quad K_{z}(w)=K(w, z) .
$$

From the estimates for Bergman kernel function given by Fefferman in [10] we have

$$
C^{-1} \delta^{-n-1}(z) \leq K(z) \leq C \delta^{-n-1}(z)
$$

for some fixed constant $C$ depending only on $D$ where $\delta(z)=\operatorname{dist}(z, \partial D)$. For a holomorphic function $f$ on $D$, let

$$
\left|\nabla^{n+1} f(z)\right|=\sum_{|\alpha| \leq n+1}\left|\frac{\partial^{|\alpha|} f}{\partial z^{\alpha}}(z)\right| .
$$

For $1 \leq p \leq \infty$, we say that $f \in B^{p}(D)$ if

$$
\|f\|_{B^{p}(D)}=\left(\int_{D}\left|\nabla^{n+1} f(z)\right|^{p} \delta(z)^{p(n+1)} d \lambda(z)\right)^{1 / p}<\infty .
$$

One can show that $k_{z} \in B^{p}(D)$ and $\left\|k_{z}\right\|_{B^{p}(D)}$ is comparable to 1 for all $z \in D$ and $1 \leq p \leq \infty$, and it can be found in $[23,24]$ and references therein that if $D$ is a smoothly bounded strictly pseudoconvex domain in $\mathbb{C}^{n}$ (or more general), one has the following inclusions:

$$
B^{p}(D) \subset B^{s}(D) \subset V M O A(D) \subset B M O A(D) \subset H^{q}(D)
$$

for $1 \leq p \leq s<\infty$ and $0<q<\infty$, and

$$
\operatorname{VMOA}(D) \subset \mathcal{B}_{0}(D) \subset \mathcal{B}(D) \subset A^{q}(D)
$$

for $1 \leq p<s<\infty, 0<q<\infty$. It is well known that $B^{\infty}(D)=\mathcal{B}(D)$, the holomorphic Bloch space over $D$ while $\mathcal{B}_{0}(D)$ is the little Bloch space.

The ideas of Coifman and Rochberg in [4] and Luecking in [26] show that one can have a represention theorems for Hardy and Bergman space and VMO and little Block space. Using their approach we prove a representation theorem for Besov space over bounded strictly pseudoconvex domains in $\mathbb{C}^{n}$. To describe the representation theorem for functions in $B^{p}(D)$, let $\left\{z_{j, k}\right\}_{j=1, k=1}^{\infty, n_{j}}$ be a sequence satisfying $\epsilon$-separation and $\delta_{\epsilon}$-density conditions(see Definition 2.2 for definition) and let $\mathcal{B}^{p}(D)$ be spaces of all holomorphic functions $f(z)$ having the representations:

$$
f(z)=\sum_{j=1}^{\infty} \sum_{k=1}^{n_{j}} c_{j, k} k_{z_{j, k}}(z), \quad\left\{c_{j, k}\right\}_{j=1, k=1}^{\infty, n_{j}} \in \ell^{p}
$$


with the norm

$$
\|f\|_{\mathcal{B}^{p}(D)}=\inf \left\{\left\|\left\{c_{j, k}\right\}\right\|_{\ell^{p}}: f(z)=\sum_{j=1}^{\infty} \sum_{k=1}^{n_{j}} c_{j, k} k_{z_{j, k}}(z)\right\} .
$$

We first prove the following representation theorem for functions in Besov spaces.

THEOREM 1.1. Let $D$ be a smoothly bounded strictly pseudoconvex domain in $\mathbb{C}^{n}$ and $1 \leq p<\infty$. Then, there is a sequence of points $\left\{z_{j, k}\right\}_{j=1, k=1}^{\infty, n_{j}}$ satisfying $\epsilon$-separation and $\delta_{\epsilon}$-density conditions so that

$$
\left(B^{p}(D),\|\cdot\|_{B^{p}}\right)=\left(\mathcal{B}^{p}(D),\|\cdot\|_{\mathcal{B}^{p}}\right)
$$

with norms equivalent.

We apply this representation theorem to composition operators on Besov spaces. First, we introduce some notation. For $1 \leq p \leq \infty$ and for each $z \in D$, let

$$
M_{\phi, p}(z)=\left\|C_{\phi}\left(k_{z}\right)\right\|_{B^{p}(D)} .
$$

For any $\epsilon>0$, let

$$
D_{\epsilon}=\{z \in D: \operatorname{dist}(z, \partial D)>\epsilon\} \quad \text { and } \quad D_{\epsilon}^{c}=D \backslash D_{\epsilon} .
$$

As applications of Theorem 1.1, we prove the following.

THEOREM 1.2. Let $D$ be a smoothly bounded strictly pseudoconvex domain in $\mathbb{C}^{n}$ and let $\phi$ be a holomorphic self map on D. Then,

(1) $C_{\phi}$ is bounded on $B^{1}(D)$ if and only if $M_{\phi, 1}(z) \in L^{\infty}(D)$;

(2) $C_{\phi}$ is compact on $B^{1}(D)$ if and only if $M_{\phi, 1}(z) \in C_{0}(D)$;

(3) If $C_{\phi}$ is bounded on $B^{1}(D)$, then $C_{\phi}$ is bounded on $B^{p}(D)$ for $1<p<\infty$;

(4) If $C_{\phi}$ is compact on $B^{1}(D)$ then $C_{\phi}$ is compact on $B^{p}(D)$ for $1<p<\infty$;

(5) If $M_{\phi, p} \in L^{p^{\prime}}(D, d \lambda)$ for $1<p<\infty$, then $C_{\phi}$ is bounded on $B^{p}(D)$;

(6) If $\lim _{\epsilon \rightarrow 0^{+}}\left\|M_{\phi, p}\right\|_{L^{p^{\prime}}\left(D_{\epsilon}^{c}, d \lambda\right)}=0$ for $1<p<\infty$, then $C_{\phi}$ is compact on $B^{p}(D)$.

The paper is organized as follows. In Section 2, we provide some basic results on strictly convex domains in $\mathbb{C}^{n}$, and as a consequence we prove Corollary 2.6 which gives one directional inclusion for Theorem 1.1. In Section 3, we prove duality theorems for Besov space $B^{p}(D)$ with an appropriate paring, and complete our proof of Theorem 1.1. Theorem 1.2 is proved in Section 5 .

Constants. In the rest of the paper we use the same letter $C$ to denote various positive constants which may change at each occurrence. Variables indicating the dependency of constants $C$ will be often specified in the parenthesis or as a subscript. We use the notation $X \lesssim Y$ or $Y \gtrsim X$ for nonnegative quantities $X$ and $Y$ to mean $X \leq C Y$ for some inessential constant $C>0$. Similarly, we use the notation $X \approx Y$ if both $X \lesssim Y$ and $Y \lesssim X$ hold.

2. Preliminary. For the rest of the paper we let $D$ be a fixed domain in $\mathbb{C}^{n}$ which is smoothly bounded and strictly pseudoconvex. Let $\delta(z):=\operatorname{dist}(z, \partial D)$ be the distance function from $z$ to $\partial D$. For each $z \in D$ near $\partial D$, let $\nu(z)=\left(\frac{\partial \delta(z)}{\partial \bar{z}_{1}}, \cdots, \frac{\partial \delta(z)}{\partial \bar{z}_{n}}\right)$, the complex normal vector at $z$ of $\partial D_{\delta(z)}$. The orthogonal complement of $\nu(z)$ in $\mathbb{C}^{n}$ is

$$
\mathbb{C}_{\nu(z)}^{n-1}=\left\{w \in \mathbb{C}^{n}:\langle w, \nu(z)\rangle=0\right\}
$$


Let $\left\{w_{1, z}, \cdots, w_{n-1, z}\right\}$ be an orthonormal basis for $\mathbb{C}_{\nu(z)}^{n-1}$. Then a non-isotropic polydisc (or ball) in $D$ centered at $z \in D$ and radius $\epsilon>0$ is defined as follows:

$$
E_{\epsilon}(z)=\left\{\sum_{j=1}^{n-1} \lambda_{j} w_{j, z}+\lambda_{n} \nu(z):\left|\lambda_{j}\right|^{2}<\epsilon^{2} \delta(z),\left|\lambda_{n}\right| \leq \epsilon \delta(z), 1 \leq j<n\right\} .
$$

Since $D$ is strictly pseudoconvex with $C^{2}$ boundary, there are two positive numbers $\epsilon_{D}$ and $C_{D}$ so that if $0<\epsilon \leq \epsilon_{D}$, then $E_{2 \epsilon}(z) \subset D$ and

(i) if $0<r_{1}<r_{2}$, then $E_{r_{1}}(z) \subset E_{r_{2}}(z)$,

(ii) if $E_{r_{1}}(z) \cap E_{r_{2}}(w) \neq \emptyset$ with $r_{1} \leq r_{2}$, then $E_{r_{1}}(z) \subset E_{C_{D} r_{2}}(w)$,

(iii) $\delta(w) \approx \delta(z)$ for $w \in E_{\epsilon}(z)$.

Applying the idea of the Whitney Covering Lemma in [1] and decomposition of $D$ in [4] for bounded symmetric domain in $\mathbb{C}^{n}$ and the unit ball of $\mathbb{C}^{n}$ in [26], one has the following lemma.

Lemma 2.1. There is $c>0$ depending only on $D$ so that for any $\epsilon>0$ with $\delta_{\epsilon}=: c \epsilon<\epsilon_{D} / 2$, there is a sequence of points $\left\{z_{j, k}\right\}_{k=1, j=1}^{n_{j}, \infty}$ of $D$ satisfying

$$
\delta\left(z_{j, k}\right)=\delta\left(z_{j, m}\right), \quad \frac{\epsilon}{c 2^{j}} \leq \delta\left(z_{j, k}\right) \leq \frac{c}{\epsilon 2^{j}}, \quad 1 \leq k, m \leq n_{j}, j=1,2,3, \cdots,
$$

and

$$
\left\{E_{\epsilon}\left(z_{j, k}\right)\right\}_{k=1, j=1}^{n_{j}, \infty} \text { are disjoint and } D=\cup_{j=1}^{\infty} \cup_{k=1}^{n_{j}} E_{c \epsilon}\left(z_{j, k}\right)
$$

Note that by (2.2) and (2.3) we have

$$
n_{j} \approx \delta\left(z_{j, k}\right)^{-n}, \quad 1 \leq k \leq n_{j}, \quad j=1,2,3, \cdots .
$$

Definition 2.2. A sequence $\left\{z_{j, k}\right\}_{k=1, j=1}^{n_{j}, \infty}$ of points in $D$ is said to be satisfying $\epsilon$-separation and $\delta_{\epsilon^{-}}$density conditions if it satisfies (2.2) and (2.3).

For any positive defining function $r(z)$ for $D$ with $r \in C^{2}(\bar{D})$, denote by $K_{r}(z, w)$, the Bergman kernel function for the weighted Bergman space $A^{2}\left(D, r(z)^{n+1} d v\right)$. Let

$$
V_{r}(f)(z)=r(z)^{n+1} \int_{D} f(w) K_{r}(z, w) d v(w), \quad z \in D
$$

and let $P$ be the Bergman projection defined as

$$
P(f)(z)=\int_{D} f(w) K(z, w) d v(w), \quad z \in D .
$$

Proposition 2.3. For $1 \leq p<\infty$, we have

(1) $V_{r} P=V_{r}$ on $L^{p}$;

(2) $V_{r}\left(K_{\xi}\right)(z)=r(z)^{n+1} K_{r}(z, \xi)$. 
Proof. For any $f \in C_{0}^{\infty}(\bar{D})$, one has

$$
\begin{aligned}
V_{r}(P(f))(z) & =r(z)^{n+1} \int_{D} K_{r}(z, w) P(f)(w) d v(w) \\
& =r(z)^{n+1} \int_{D} K_{r}(z, w) \int_{D} f(\xi) K(w, \xi) d v(\xi) d v(w) \\
& =r(z)^{n+1} \int_{D} f(\xi) \int_{D} K_{r}(z, w) K(w, \xi) d v(w) d v(\xi) \\
& =r(z)^{n+1} \int_{D} f(\xi) K_{r}(z, \xi) d v(\xi) \\
& =V_{r}(f)(z) .
\end{aligned}
$$

Thus, (1) follows from this since $C_{0}^{\infty}(\bar{D})$ is dense in $L^{p}$.

For (2) note that for $\xi \in D$

$$
\begin{aligned}
V_{r}\left(K_{\xi}\right)(z) & =r(z)^{n+1} \int_{D} K_{r}(z, w) K_{\xi}(w) d v(w) \\
& =r(z)^{n+1} K_{r}(z, \xi) .
\end{aligned}
$$

The following is proved by Li and Luo in [23]:

TheOREM 2.4. For $1 \leq p<\infty$, we have

(1) $P\left(L^{p}(D, d \lambda)\right)=B^{p}(D)$;

(2) $V_{r}: B^{p}(D) \rightarrow L^{p}(D, d \lambda)$ is bounded;

(3) $P V_{r}=I$ on $B^{p}(D)$.

With Theorem 2.4 at hand, we prove the following proposition from which we can easily deduce one implication of Theorem 1.1 .

Proposition 2.5. Let $\left\{z_{j, k}\right\}_{k=1, j=1}^{n_{j}, \infty}$ be a sequence on points of $D$ satisfying $\epsilon$-separation and $\delta_{\epsilon}$-density condition. Then, the following holds;

(1) For $1 \leq p<\infty$ and $\left\{c_{j, k}\right\}_{k=1, j=1}^{n_{j}, \infty} \in \ell^{p}$, we have

$$
f(z):=\sum_{j=1}^{\infty} \sum_{k=1}^{n_{j}} c_{j, k} k_{z_{j, k}}(z) \in B^{p}(D)
$$

and

$$
\|f\|_{B^{p}(D)} \lesssim\left\|\left\{c_{j, k}\right\}_{k=1, j=1}^{n_{j}, \infty}\right\|_{\ell^{p}} .
$$

(2) If $f$ is given as in (1) with $\left\{c_{j, k}\right\}_{k=1, j=1}^{n_{j}, \infty} \in c_{0}$, then $f \in \mathcal{B}_{0}(D)$.

Proof. Let

$$
F(z)=\sum_{j=1}^{\infty} \sum_{k=1}^{n_{j}} c_{j, k} K\left(z_{j, k}\right)^{-1}\left|E_{\epsilon}\left(z_{j, k}\right)\right|^{-1} \chi_{E_{\epsilon}\left(z_{j, k}\right)}(z) .
$$

Since $\left\{E_{\epsilon}\left(z_{j, k}\right)\right\}_{k=1, j=1}^{n_{j}, \infty}$ are disjoint and $\delta(w) \approx \delta(z)$ for $w \in E_{\epsilon}(z)$, we have

$$
\begin{aligned}
\int_{D}|F(z)|^{p} d \lambda(z) & =\sum_{j=1}^{\infty} \sum_{k=1}^{n_{j}}\left|c_{j, k}\right|^{p}\left(K\left(z_{j, k}\right)^{-1}\left|E_{\epsilon}\left(z_{j, k}\right)\right|^{-1}\right)^{p} \int_{E_{\epsilon}\left(z_{j k}\right)} d \lambda(z) \\
& \lesssim \epsilon^{2 n(1-p)} \sum_{j=1}^{\infty} \sum_{k=1}^{n_{j}}\left|c_{j, k}\right|^{p}
\end{aligned}
$$


and

$$
\begin{aligned}
P(F)(z) & =\sum_{j=1}^{\infty} \sum_{k=1}^{n_{j}} c_{j, k} K\left(z_{j, k}\right)^{-1}\left|E_{\epsilon}\left(z_{j, k}\right)\right|^{-1} \int_{E_{\epsilon}\left(z_{j, k}\right)} K(z, w) d v(w) \\
& =\sum_{j=1}^{\infty} \sum_{k=1}^{n_{j}} c_{j, k} k_{z_{j, k}}(z) \\
& =f(z) .
\end{aligned}
$$

Therefore, by (1) of Theorem 2.4, we get

$$
\|f\|_{B^{p}(D)}=\|P(F)\|_{B^{p}(D)} \lesssim\|F\|_{L^{p}(D, d \lambda)} \lesssim\left(\epsilon^{2 n(1-p)} \sum_{j=1}^{\infty} \sum_{k=1}^{n_{j}}\left|c_{j, k}\right|^{p}\right)^{1 / p}
$$

which proves (1).

To prove (2), let

$$
F_{m}(z)=\sum_{j=1}^{m} \sum_{k=1}^{n_{j}} c_{j, k} K\left(z_{j, k}\right)^{-1}\left|E_{\epsilon}\left(z_{j, k}\right)\right|^{-1} \chi_{E_{\epsilon}\left(z_{j, k}\right)}(z) .
$$

Since $\left\{c_{j, k}\right\}_{k=1, j=1}^{n_{j}, \infty} \in c_{0}$, we get

$$
\lim _{m \rightarrow \infty}\left\|F_{m}(z)-F(z)\right\|_{L^{\infty}(D)}=0 .
$$

Also, note that from the estimates of the derivatives of the Bergman kernel we get

$$
\begin{aligned}
\left\|f-P\left(F_{m}\right)\right\|_{\mathcal{B}(D)} & \lesssim \sup _{z \in D} \delta(z) \int_{D}\left|\nabla_{z} K(z, w) \| F(w)-F_{m}(w)\right| d v(w) \\
& \lesssim\left\|F-F_{m}\right\|_{L^{\infty}(D)} .
\end{aligned}
$$

Therefore, we have

$$
\lim _{m \rightarrow \infty}\left\|f-P\left(F_{m}\right)\right\|_{\mathcal{B}(D)} \lesssim \lim _{m \rightarrow \infty}\left\|F-F_{m}\right\|_{L^{\infty}(D)}=0
$$

Hence, we get $f=P(F) \in \mathcal{B}_{0}(D)$ since $P\left(F_{m}\right) \in C^{\infty}(\bar{D})$.

As a corollary we get a proof for one directional inclusion of Theorem 1.1

Corollary 2.6. For $1 \leq p<\infty$, we have

$$
\mathcal{B}^{p}(D) \subset B^{p}(D)
$$

and

$$
\|f\|_{B^{p}(D)} \lesssim\|f\|_{\mathcal{B}^{p}(D)}
$$


3. Duality and Decomposition for $B^{p}(D)$. In this section, through certain relations between $B^{p}$ and $\ell^{p}$ we prove the duality theorem for $B^{p}$ and complete the proof of Theorem 1.1. We follow the scheme of Coifman and Rochberg in [4] using Theorem 2.4.

Consider a linear operator $T: B^{p}(D) \rightarrow \ell^{p}$ defined as follows: For $f \in B^{p}(D)$, let

$$
T(f)=\left\{V_{r}(f)\left(z_{j, k}\right)\right\}_{k=1, j=1}^{n_{j}, \infty}, \quad r(z)^{n+1}=K(z)^{-1} .
$$

This linear operator $T$ plays a key role in the proof of Theorem 1.1 and the following holds.

THEOREM 3.1. The following holds:

(1) $T: B^{p}(D) \rightarrow \ell^{p}$ is bounded and $\|T(f)\|_{\ell^{p}} \approx\|f\|_{B^{p}(D)}$ if $1<p<\infty$;

(2) $T: \mathcal{B}_{0}(D) \rightarrow c_{0}$ is bounded and injective.

Proof. Let $1<p<\infty$ and

$$
T_{K_{r}}(f)(z)=\int_{D} K_{r}(z, w) f(w) d v(w)
$$

Then, from the plurisubmarmonicity of $T_{K_{r}}(f)$ and the fact that $r(z) \approx r\left(z_{j, k}\right)$ on $E_{\epsilon}\left(z_{j, k}\right)$, we have

$$
\left|V_{r}(f)\left(z_{j, k}\right)\right| \leq\left|E_{\epsilon}\left(z_{j, k}\right)\right|^{-1} \int_{E_{\epsilon}\left(z_{j, k}\right)}\left|V_{r}(f)(z)\right| d v(z) .
$$

Since $\left\{E_{\epsilon}\left(z_{j, k}\right)\right\}_{k=1, j=1}^{n_{j}, \infty}$ are disjoint subsets in $D$, we thus have

$$
\begin{aligned}
\|T(f)\|_{\ell^{p}}^{p} & =\sum_{j=1}^{\infty} \sum_{k=1}^{n_{j}}\left|V_{r}(f)\left(z_{j, k}\right)\right|^{p} \\
& \leq \sum_{j=1}^{\infty} \sum_{k=1}^{n_{j}} \frac{1}{\left|E_{\epsilon}\left(z_{j, k}\right)\right|} \int_{E_{\epsilon}\left(z_{j, k}\right)}\left|V_{r}(f)(z)\right|^{p} d v(z) \\
& \lesssim \sum_{j=1}^{\infty} \sum_{k=1}^{n_{j}} \int_{E_{\epsilon}\left(z_{j, k}\right)}\left|V_{r}(f)(z)\right|^{p} d \lambda(z) \\
& \leq \int_{D}\left|V_{r}(f)(z)\right|^{p} d \lambda(z) \\
& \lesssim\|f\|_{B^{p}(D)}^{p} .
\end{aligned}
$$

Here, the last inequality follows from (2) of Theorem 2.4. This proves that $T$ : $B^{p}(D) \rightarrow \ell^{p}$ is bounded when $1<p<\infty$.

Next, by choosing $\epsilon>0$ small enough we show that

$$
\left\|V_{r}(f)\right\|_{L^{p}(D, d \lambda)} \lesssim\|T(f)\|_{\ell^{p}}
$$

This completes proof of (1) since we have

$$
\|f\|_{B^{p}(D)} \lesssim\left\|V_{r}(f)\right\|_{L^{p}(D, d \lambda)}
$$

by (1) and (3) of Theorem 2.4. First, we choose $\epsilon>0$ small enough so that

$$
c(\epsilon):=\sup \left\{\left|E_{\delta_{\epsilon}}\left(z_{j, k}\right)\right| K(z): z \in E_{\delta_{\epsilon}}\left(z_{j, k}\right): 1 \leq k \leq n_{j}, j=1,2,3, \cdots\right\} \lesssim 1 .
$$


Choose $\xi_{j, k} \in E_{\delta_{\epsilon}}\left(z_{j, k}\right)$ so that

$$
\left|V_{r}(f)\left(\xi_{j, k}\right)\right|=\max \left\{\left|V_{r}(f)(z)\right|: z \in E_{\delta_{\epsilon}}\left(z_{j, k}\right)\right\} .
$$

Since $D \subset \cup_{j=1}^{\infty} \cup_{k=1}^{n_{j}} E_{\delta_{\epsilon}}\left(z_{j, k}\right)$, we see that

$$
\begin{aligned}
\left\|V_{r}(f)\right\|_{L^{p}(D, d \lambda)}^{p} & \leq \sum_{j=1}^{\infty} \sum_{k=1}^{n_{j}} \int_{E_{\delta_{\epsilon}}\left(z_{j, k}\right)}\left|V_{r}(f)\right|^{p} d \lambda(z) \\
& \lesssim c(\delta) \sum_{j=1}^{\infty} \sum_{k=1}^{n_{j}}\left|V_{r}(f)\left(\xi_{j, k}\right)\right|^{p} \\
& \lesssim \sum_{j=1}^{\infty} \sum_{k=1}^{n_{j}}\left|V_{r}(f)\left(z_{j, k}\right)\right|^{p}+\sum_{j=1}^{\infty} \sum_{k=1}^{n_{j}}\left|V_{r}(f)\left(\xi_{j, k}\right)-V_{r}(f)\left(z_{j, k}\right)\right|^{p} .
\end{aligned}
$$

Thus, it suffices to show that

$$
\sum_{j=1}^{\infty} \sum_{k=1}^{n_{j}}\left|V_{r}(f)\left(\xi_{j, k}\right)-V_{r}(f)\left(z_{j, k}\right)\right|^{p} \lesssim \sum_{j=1}^{\infty} \sum_{k=1}^{n_{j}}\left|V_{r}(f)\left(z_{j, k}\right)\right|^{p}
$$

From (1) of Theorem 2.4 we can choose $f_{0} \in L^{p}(D, d \lambda)$ so that

$$
\left\|f_{0}\right\|_{L^{p}(D, d \lambda)} \approx\|f\|_{B^{p}(D)} \quad \text { and } f=P\left(f_{0}\right) .
$$

Let

$$
A\left(z_{j, k}, \xi_{j, k}\right)=\int_{D}\left|K_{r}\left(z_{j, k}, w\right)-K_{r}\left(\xi_{j, k}, w\right)\right| d v(w)
$$

If $f$ is holomorphic, then $f(a)-f(b)=\langle\nabla f(a+t(b-a)), \overline{a-b}\rangle$ for for some $t \in[0,1]$.

Thus, from the estimates of the (weighted) Bergman kernel we have

$$
\left|K\left(z_{j, k}\right)-K\left(\xi_{j, k}\right)\right| \lesssim \epsilon K\left(z_{j, k}\right) \quad \text { and } \quad A\left(z_{j, k}, \xi_{j, k}\right) \lesssim \epsilon K\left(z_{j, k}\right) .
$$

Since $V_{r} P=V_{r}$ by (1) of Proposition 2.3, we then have

$$
\begin{aligned}
& \left|V_{r}(f)\left(\xi_{j, k}\right)-V_{r}(f)\left(z_{j, k}\right)\right| \\
& =\left|V_{r}\left(f_{0}\right)\left(\xi_{j, k}\right)-V_{r}\left(f_{0}\right)\left(z_{j, k}\right)\right| \\
& =\left|K\left(\xi_{j, k}\right)^{-1} T_{K_{r}}\left(f_{0}\right)\left(\xi_{j, k}\right)-K\left(z_{j, k}\right)^{-1} T_{K_{r}}\left(f_{0}\right)\left(z_{j, k}\right)\right| \\
& \leq\left|\left(K\left(\xi_{j, k}\right)^{-1}-K\left(z_{j, k}\right)^{-1}\right) T_{K_{r}}\left(f_{0}\right)\left(z_{j, k}\right)\right| \\
& \quad+K\left(\xi_{j, k}\right)^{-1}\left|T_{K_{r}}\left(f_{0}\right)\left(\xi_{j, k}\right)-T_{K_{r}}\left(f_{0}\right)\left(z_{j, k}\right)\right| \\
& \quad \lesssim \quad K\left(z_{j, k}\right)^{-1}\left|T_{K_{r}}\left(f_{0}\right)\left(z_{j, k}\right)\right| \\
& \quad+K\left(\xi_{j, k}\right)^{-1} \int_{D}\left|K_{r}\left(\xi_{j, k}, w\right)-K_{r}\left(z_{j, k}, w\right)\right|\left|f_{0}(w)\right| d v(w) \mid \\
& \quad \\
& \quad \lesssim \epsilon\left|V_{r}(f)\left(z_{j, k}\right)\right|+K\left(\xi_{j, k}\right)^{-1} \int_{D}\left|K_{r}\left(\xi_{j, k}, w\right)-K_{r}\left(z_{j, k}, w\right)\right|\left|f_{0}(w)\right| d v(w) \mid .
\end{aligned}
$$


Also, note that by Hölder's inequality we get

$$
\begin{aligned}
& \left(\left|K\left(z_{j, k}\right)^{-1} \int_{D}\right| K_{r}\left(\xi_{j, k}, w\right)-K_{r}\left(z_{j, k}, w\right)|| f_{0}(w)|d v(w)|\right)^{p} \\
& \left.\leq K\left(z_{j, k}\right)^{-p^{\prime}} A\left(z_{j, k}, \xi_{j, k}\right)^{p^{\prime}-1} \int_{D} \mid K_{r}\left(\xi_{j, k}, w\right)-K_{r}\left(z_{j, k}, w\right)\right)\left.|| f_{0}(w)\right|^{p} d v(w) \\
& \left.\lesssim \epsilon K\left(z_{j, k}\right)^{-1} \int_{D} \mid K_{r}\left(\xi_{j, k}, w\right)-K_{r}\left(z_{j, k}, w\right)\right)\left.|| f_{0}(w)\right|^{p} d v(w) \\
& \left.\lesssim \epsilon K\left(z_{j, k}\right)^{-1} \int_{D} \mid K_{r}\left(z_{j, k}, w\right)\right)\left.|| f_{0}(w)\right|^{p} d v(w) \\
& \left.\lesssim \epsilon \int_{D} \int_{E_{\delta_{\epsilon}}\left(z_{j, k}\right)} \mid K_{r}\left(z_{j, k}, w\right)\right)\left.|| f_{0}(w)\right|^{p} d v(z) d v(w) .
\end{aligned}
$$

Therefore, we have

$$
\begin{aligned}
& \sum_{j=1}^{\infty} \sum_{k=1}^{n_{j}}\left|V_{r}(f)\left(\xi_{j, k}\right)-V_{r}(f)\left(z_{j, k}\right)\right|^{p} \\
& \left.\lesssim \epsilon \sum_{j=1}^{\infty} \sum_{k=1}^{n_{j}}\left|V_{r}(f)\left(z_{j, k}\right)\right|^{p}+\epsilon \sum_{j=1}^{\infty} \sum_{k=1}^{n_{j}} K\left(z_{j, k}\right)^{-1} \int_{D} \mid K_{r}\left(z_{j, k}, w\right)\right)\left.|| f_{0}(w)\right|^{p} d v(w) \\
& \left.\lesssim \epsilon \sum_{j=1}^{\infty} \sum_{k=1}^{n_{j}}\left|V_{r}(f)\left(z_{j, k}\right)\right|^{p}+\epsilon \int_{D} \int_{D} \mid K_{r}(z, w)\right)\left.|| f_{0}(w)\right|^{p} d v(w) d v(z) \\
& \lesssim \epsilon \sum_{j=1}^{\infty} \sum_{k=1}^{n_{j}}\left|V_{r}(f)\left(z_{j, k}\right)\right|^{p}+\epsilon \int_{D}\left|f_{0}(w)\right|^{p} d \lambda(z) d \lambda(w) .
\end{aligned}
$$

By (3.3) and (3.5), we then get (3.4) and hence completes proof of (1).

To prove (2), first note that $\left\|V_{r}(f)\right\|_{L^{\infty}} \lesssim\|f\|_{L^{\infty}}$ which implies $T: B^{\infty}(D) \rightarrow \ell^{\infty}$ is bounded. Thus, for any $f \in \mathcal{B}_{0}(D)$ we have $T(f) \in \ell^{\infty}$. It is known from [14] that there is an $f_{0} \in C_{0}(D)$ so that $P\left(f_{0}\right)=f$ and $\left\|f_{0}\right\|_{C(\bar{D})} \approx\|f\|_{\mathcal{B}_{0}(D)}$. Thus for any $\epsilon>0$, there is $\delta>0$ so that

$$
\left|f_{0}(z)\right| \leq \epsilon, \text { when } z \in D \backslash D_{\delta}=\{w \in D: \delta(w) \leq \delta\} .
$$

By (1) of Proposition 2.3, we have

$$
V_{r}(f)(z)=V_{r}\left(P\left(f_{0}\right)\right)(z)=V_{r}\left(f_{0}\right)(z)=r(z)^{n+1} T_{K_{r}}\left(f_{0}\right)(z) .
$$

Therefore,

$$
\begin{aligned}
& \left|V_{r}(f)\left(z_{j, k}\right)\right| \\
& =r\left(z_{j, k}\right)^{n+1}\left|\int_{D} f_{0}(w) K_{r}\left(z_{j, k}, w\right) d v(w)\right| \\
& \leq r\left(z_{j, k}\right)^{n+1}\left[\left|\int_{D_{\delta}} f_{0}(w) K_{r}\left(z_{j, k}, w\right) d v(w)\right|+\left|\int_{D \backslash D_{\delta}} f_{0}(w) K_{r}\left(z_{j, k}, w\right) d v(w)\right|\right] \\
& \lesssim r\left(z_{j, k}\right)^{n+1}\left[\left\|f_{0}\right\|_{C(\bar{D})} \delta^{-2(n+1)}+\epsilon r\left(z_{j, k}\right)^{-n-1}\right] .
\end{aligned}
$$

Therefore, we see that

$$
\lim _{j \rightarrow \infty}\left|V_{r}(f)\left(z_{j, k}\right)\right|=0 .
$$


This implies that $T(f) \in c_{0}$. With a similar argument, one has $T: \mathcal{B}_{0}(D) \rightarrow c_{0}$ is injective and proof of (2) is complete.

For $1 \leq p<\infty$ let $p^{\prime}$ be the conjugate of $p$ defined by the relation $1 / p+1 / p^{\prime}=1$.

THEOREM 3.2. With the following pairing for functions in $B^{p}(D)$,

$$
(f, g)_{0}=\int_{D} V_{r}(f)(z) \overline{V_{r}(g)(z)} d \lambda(z)
$$

we have the following duality relations:

(1) $B^{p}(D)^{*}=B^{p^{\prime}}(D)$ for $1 \leq p<\infty$;

(2) $\mathcal{B}_{0}(D)^{*}=B^{1}(D)$.

Proof. We first prove (1). It is obvious that $B^{p^{\prime}}(D) \subset\left(B^{p}(D)\right)^{*}$ with respect to the pairing (3.7) since $V_{r}: B^{p}(D) \rightarrow L^{p}(D, d \lambda)$ is bounded for $1 \leq p<\infty$ by (2) of Theorem 2.4 which is also true for $p=\infty$ from the estimate of the weighted Bergman kernel. On the other hand, if $\mathcal{L}$ is a bounded linear functional on $B^{p}(D)$ with respect to (3.7), which is interpreted as that $\mathcal{L}$ is a bounded linear functional on the subspace $\left\{V_{r}(f): f \in B^{p}(D)\right\}$ of $L^{p}(D, d \lambda)$. By Hahn Banach theorem, $\mathcal{L}$ can be extended as a bounded linear functional on $L^{p}(D, d \lambda)$ with the same norm. Since $L^{p}(D)^{*}=L^{p^{\prime}}(D)$, there is a $g \in L^{p^{\prime}}(D, d \lambda)$ so that

$$
\mathcal{L}(f)=\int_{D} V_{r}(f)(z) \overline{g(z)} d \lambda(z), \quad\|\mathcal{L}\|=\|g\|_{L^{p}(D, d \lambda)}
$$

for all $f \in B^{p}(D)$. Since $r(z)^{n+1} d \lambda=d v(z)$ and $P V_{r}=I$, we have

$$
\begin{aligned}
\mathcal{L}(f) & =\int_{D} T_{K_{r}}(f)(z) \bar{g}(z) d v(z) \\
& =\int_{D} T_{K_{r}}(f)(z) \overline{P(g)(z)} d v(z) \\
& =\int_{D} f(w) \overline{T_{K_{r}}(P(g))}(w) d v(w) \\
& =\int_{D} P V_{r}(f)(w) \overline{T_{K_{r}}(P(g))}(w) d v(w) \\
& =\int_{D} V_{r}(f)(w) \overline{T_{K_{r}}(P(g))}(w) d v(w) \\
& =\int_{D} V_{r}(f)(w) \overline{V_{r}(P(g))(w)} d \lambda(w) .
\end{aligned}
$$

Since $P: L^{p}(D, d \lambda) \rightarrow B^{p}(D)$ is bounded for $1 \leq p \leq \infty$, we have $P(g) \in B^{p^{\prime}}(D)$ and $\mathcal{L}$ can be identified as $P(g)$. The proof of (1) is complete.

Next we prove (2). By (1) with $p=1$, we have $B^{1}(D)^{*}=\mathcal{B}(D)$ with respect to the pairing (3.7). Thus, one can easily see that $B^{1}(D) \subset \mathcal{B}_{0}(D)^{*}$. On the other hands, for any linear functional $\mathcal{L}$ on $\mathcal{B}_{0}(D)$ with respect to the pairing $(3.7)$, which means that $\mathcal{L}$ is viewed as a bounded linear functional on the linear subspace $\left\{V_{r}(f): f \in \mathcal{B}_{0}(D)\right\}$ of $C_{0}(D)$. By Hahn-Banach theorem, $\mathcal{L}$ can be extended as a linear functional on $C_{0}(D)$ with the same norm. By Riesz representation theorem, there is a finite complex Borel measure $d \mu$ on $D$ so that

$$
\mathcal{L}(g)=\int_{D} V_{r}(g)(z) d \mu(z)=\int_{D} V_{r}(g)(w) \overline{P\left(r^{n+1} \overline{d \mu}\right)(w)} d \lambda,
$$


where

$$
f(z):=P\left(r^{n+1} \overline{d \mu}\right)(z)=\int_{D} K(z, w) r(w)^{n+1} d \bar{\mu}(w)
$$

Then, following the arguments of the proof of (1) we get

$$
\begin{aligned}
\mathcal{L}(g)=\int_{D} V_{r}(g)(z) \overline{f(z)} d \lambda(z) & =\int_{D} V_{r}(g)(z) \overline{V_{r}(f)(z)} d \lambda(z) \\
& =\int_{D} V_{r}(g)(z) \overline{V_{r} P\left[V_{r}(f)\right](z)} d \lambda(z) .
\end{aligned}
$$

Notice that

$$
V_{r}(f)(z)=r(z)^{n+1} \int_{D} K_{r}(z, w) f(w) d v(w)=r(z)^{n+1} \int_{D} K_{r}(z, w) r(w)^{n+1} d \bar{\mu}(w)
$$

and thus

$$
\begin{aligned}
\int_{D}\left|V_{r}(f)(z)\right| d \lambda(z) & =\int_{D}\left|\int_{D} K_{r}(z, w) r(w)^{n+1} d \bar{\mu}(w)\right| d v(z) \\
& \leq \int_{D} \int_{D}\left|K_{r}(z, w)\right| r(w)^{n+1} d v(z)|d \bar{\mu}|(w) \\
& \lesssim \int_{D} r(w)^{-n-1} r(w)^{n+1}|d \bar{\mu}|(w) \\
& \lesssim\|d \mu\| .
\end{aligned}
$$

This implies that $V_{r}(f) \in L^{1}(D, d \lambda)$, so $P V_{r}(f) \in B^{1}(D)$ with $\left\|P V_{r}(f)\right\|_{B^{1}(D)} \lesssim$ $\|d \mu\|$. Therefore, $\mathcal{B}_{0}(D)^{*}=B^{1}(D)$, the proof of $(2)$ is complete, and so is Theorem 3.2. ㅁ

We now are ready to complete the proof for Theorem 1.1.

Proof of Theorem 1.1. By Corollary 2.6, it suffices to show that for $1 \leq p<\infty$,

$$
B^{p}(D) \subset \mathcal{B}^{p}(D)
$$

and

$$
\|f\|_{\mathcal{B}^{p}(D)} \lesssim\|f\|_{B^{p}(D)}
$$

First, we prove the case $1<p<\infty$. Let $T: B^{p^{\prime}}(D) \rightarrow \ell^{p^{\prime}}$ be a linear operator defined by (3.1). By (1) of Theorem 3.1, we see that $T: B^{p^{\prime}}(D) \rightarrow \ell^{p^{\prime}}$ is isomorphic to a subspace of $\ell^{p^{\prime}}$. Therefore, $T^{*}: \ell^{p} \rightarrow B^{p}(D)$ is a bounded and onto linear operator. 
We will evaluate $T^{*}(\alpha)(z)$ for $\alpha=\left\{\lambda_{j, k}\right\}_{k=1, j=1}^{n_{j}, \infty} \in \ell^{p^{\prime}}$. For any $g \in B^{p^{\prime}}(D)$, we have

$$
\begin{aligned}
\left(T^{*}(\alpha), g\right)_{0}= & (\alpha, T(g)) \\
& =\sum_{j=1}^{\infty} \sum_{k=1}^{n_{j}} \lambda_{j, k} \overline{V_{r}(g)\left(z_{j, k}\right)} \\
& =\sum_{j=1}^{\infty} \sum_{k=1}^{n_{j}} \lambda_{j, k} r\left(z_{j, k}\right)^{n+1} \overline{T_{K_{r}}(g)\left(z_{j, k}\right)} \\
& =\sum_{j=1}^{\infty} \sum_{k=1}^{n_{j}} \lambda_{j, k} r\left(z_{j, k}\right)^{n+1} \int_{D} \overline{T_{K_{r}}(g)(w)} K_{r}\left(w, z_{j, k}\right) r(w)^{n+1} d v(w) \\
& =\int_{D} \sum_{j=1}^{\infty} \sum_{k=1}^{n_{j}} \lambda_{j, k} r\left(z_{j, k}\right)^{n+1} r(w)^{n+1} K_{r}\left(w, z_{j, k}\right) \overline{T_{K_{r}}(g)(w)} d v(w) \\
& =\int_{D} \sum_{j=1}^{\infty} \sum_{k=1}^{n_{j}} \lambda_{j, k} r\left(z_{j, k}\right)^{n+1} r(w)^{n+1} K_{r}\left(w, z_{j, k}\right) \overline{V_{r}(g)(w)} d \lambda(w) .
\end{aligned}
$$

Let

$$
f(w)=\sum_{j=1}^{\infty} \sum_{k=1}^{n_{j}} \lambda_{j, k} r\left(z_{j, k}\right)^{n+1} K\left(w, z_{j, k}\right)
$$

then

$$
\begin{aligned}
V_{r}(f)(z) & =r(z)^{n+1} \int_{D} f(w) K_{r}(z, w) d v(w) \\
& =r(z)^{n+1} \sum_{j=1}^{\infty} \sum_{k=1}^{n_{j}} \lambda_{j, k} r\left(z_{j, k}\right)^{n+1} K_{r}\left(z, z_{j, k}\right) .
\end{aligned}
$$

Therefore, for any $g \in B^{p^{\prime}}(D)$ we have

$$
\begin{aligned}
\left(T^{*}(\alpha), g\right)_{0} & =\int_{D} \sum_{j=1}^{\infty} \sum_{k=1}^{n_{j}} \lambda_{j, k} r\left(z_{j, k}\right)^{n+1} r(w)^{n+1} K_{r}\left(w, z_{j, k}\right) \overline{V_{r}(g)(w)} d \lambda(w) \\
& =\int_{D} V_{r}(f)(w) \overline{V_{r}(g)(w)} d \lambda(w) \\
& =(f, g)_{0} .
\end{aligned}
$$

This implies that

$$
T^{*}\left(\left\{\lambda_{j, k}\right\}_{k=1, j=1}^{n_{j}, \infty}\right)(z)=f(z)=\sum_{j=1}^{\infty} \sum_{k=1}^{n_{j}} \lambda_{j, k} k_{z_{j, k}}(z) .
$$

This implies that $f \in \mathcal{B}^{p}(D)$. Since $T^{*}: \ell^{p} \rightarrow B^{p}(D)$ is onto, we have $B^{p}(D) \subset$ $\mathcal{B}^{p}(D)$. Since $T: B^{p^{\prime}}(D) \rightarrow \ell^{p^{\prime}}$ is isomorphic to a subspace of $\ell^{p^{\prime}}$, we see that

$$
\|f\|_{\mathcal{B}^{p}(D)} \lesssim\|f\|_{B^{p}(D)}
$$

This completes proof for $1<p<\infty$. 
Finally, suppose $p=1$. Since $T: \mathcal{B}_{0}(D) \rightarrow c_{0}$ is bounded and injective. Since $\mathcal{B}_{0}(D)^{*}=B^{1}(D)$ by $(2)$ of Theorem 3.2 and $\left(c_{0}\right)^{*}=\ell^{1}$, we see that $T^{*}: \ell^{1} \rightarrow B^{1}(D)$ is bounded and onto. Then, following the same argument for the case $1<p<\infty$, we can see that for any $f \in B^{1}(D)$, there is $\left\{c_{j, k}\right\}_{k=1, j=1}^{n_{j}, \infty} \in \ell^{1}$ so that

$$
f(z)=\sum_{j=1}^{\infty} \sum_{k=1}^{n_{j}} c_{j, k} k_{z_{j, k}}(z) \quad \text { with } \quad\|f\|_{B^{1}(D)} \approx \sum_{j=1}^{\infty} \sum_{k=1}^{n_{j}}\left|c_{j, k}\right| .
$$

From this we can deduce

$$
\|f\|_{\mathcal{B}^{1}(D)} \lesssim\|f\|_{B^{1}(D)}
$$

which completes proof for $p=1$, and proof of Theorem 1.1 is complete.

4. Composition operators on $B^{p}(D)$. In this section, we prove Theorem 1.2. For $1 \leq p<\infty$, let

$$
M_{p}(\phi):=\sup \left\{\left\|C_{\phi}\left(k_{z_{j, k}}\right)\right\|_{B^{p}(D)}: 1 \leq k \leq n_{j}, 1 \leq j<\infty\right\} .
$$

Throughout this section, we assume $\phi: D \rightarrow D$ is a holomorphic.

We start with the case $p=1$.

THEOREM 4.1. For any holomorphic mapping $\phi: D \rightarrow D$, we have

(1) $C_{\phi}$ is bounded on $B^{1}(D)$ if and only if

$$
M_{1}(\phi)<\infty
$$

(2) $C_{\phi}$ is compact on $B^{1}(D)$ if and only if

$$
\lim _{j \rightarrow \infty} \sup \left\{\left\|C_{\phi}\left(k_{z_{j, k}}\right)\right\|_{B^{1}(D)}: 1 \leq k \leq n_{j}\right\}=0 .
$$

Proof. Since $\left\|k_{z_{j, k}}\right\|_{B^{1}(D)} \approx 1$, the necessity condition for (1) or (2) is obvious. Thus, it is enough to prove the sufficiency condition.

For (1), suppose $M_{1}(\phi)<\infty$ and let

$$
f(z)=\sum_{j=1}^{\infty} \sum_{k=1}^{n_{j}} \lambda_{j, k} k_{z_{j, k}}(z) \quad \text { with }\|f\|_{\mathcal{B}^{1}(D)}=\sum_{j=1}^{\infty} \sum_{k=1}^{n_{j}}\left|\lambda_{j, k}\right|<\infty .
$$

Then, we have

$$
\left\|C_{\phi}(f)\right\|_{B^{1}(D)} \leq \sum_{j=1}^{\infty} \sum_{k=1}^{n_{j}}\left|\lambda_{j, k}\right|\left\|C_{\phi}\left(k_{z_{j, k}}\right)\right\|_{B^{1}(D)} \leq M_{1}(\phi)\|f\|_{\mathcal{B}^{1}(D)} .
$$

To prove (2), suppose

$$
\lim _{j \rightarrow \infty} \sup \left\{\left\|C_{\phi}\left(k_{z_{j, k}}\right)\right\|_{B^{1}(D)}: 1 \leq k \leq n_{j}\right\}=0 .
$$

To show the compactness of $C_{\phi}$ on $B^{1}(D)$, it suffices to show that $\lim _{s \rightarrow \infty}\left\|C_{\phi}\left(f_{s}\right)\right\|_{B^{1}(D)}=0$ for all sequence $\left\{f_{s}\right\}_{s=1}^{\infty}$ in $B^{1}(D)$ with norm 1 which converges to 0 uniformly on any compact subset of $D$. Let $\left\{f_{s}\right\}_{s=1}^{\infty}$ be a sequence in 
$B^{1}(D)$ with norm 1 which converges to 0 uniformly on any compact subset of $D$. Then, by Theorem 3.2

$$
f_{s}(z)=\sum_{j=1}^{\infty} \sum_{k=1}^{n_{j}} \lambda_{j, k}^{s} k_{z_{j, k}}(z), \quad \text { with }\left\|\left\{\lambda_{j . k}^{s}\right\}\right\|_{\ell^{1}} \approx 1
$$

Let $\epsilon>0$, then by assumption there is $j_{0}$ such that

$$
\left\|C_{\phi}\left(k_{z_{j}, k}\right)\right\|_{B^{1}}<\epsilon, \text { for all } 1 \leq k \leq n_{j}, j \geq j_{0} .
$$

Since $\left\{f_{s}\right\}_{s=1}^{\infty}$ converges to 0 uniformly on any compact subset of $D$, there is $s_{0}>1$ so that if $s \geq s_{0}$ then

$$
\sum_{j=1}^{j_{0}} \sum_{k=1}^{n_{j}}\left|\lambda_{j, k}^{s}\right|<\epsilon
$$

Therefore, if $s \geq s_{0}$ then

$$
\begin{aligned}
\left\|C_{\phi}\left(f_{s}\right)\right\|_{B^{1}(D)} & \leq \sum_{j=1}^{j_{0}} \sum_{k=1}^{n_{j}}\left|\lambda_{j, k}^{s}\right|\left\|C_{\phi}\left(k_{z_{j, k}}\right)\right\|_{B^{1}(D)}+\sum_{j=j_{0}+1}^{\infty} \sum_{k=1}^{n_{j}}\left|\lambda_{j, k}^{s}\right|\left\|C_{\phi}\left(k_{z_{j, k}}\right)\right\|_{B^{1}(D)} \\
& \lesssim\left\|C_{\phi}\right\| \epsilon .
\end{aligned}
$$

Therefore, $\lim _{s \rightarrow \infty}\left\|C_{\phi}\left(f_{s}\right)\right\|_{B^{1}(D)}=0$ which complete proof of $(2)$. ㅁ

Next, we consider the case when $1<p<\infty$. Let $M^{\phi}$ be the $\infty \times \infty$ matrix defined by

$$
M^{\phi}=\left[\frac{\int_{D} K\left(\phi(z), z_{j, k}\right) K_{r}\left(z_{\ell, m}, z\right) d v(z)}{K\left(z_{j, k}\right) K\left(z_{\ell, m}\right)}\right] .
$$

Then, we have the following characterization for the boundedness and the compactness which is not part of Theorem 1.2. have

TheOrem 4.2. For any holomorphic mapping $\phi: D \rightarrow D$ and $1<p<\infty$, we

(1) $C_{\phi}$ is bounded on $B^{p}(D)$ if and only if $M^{\phi}$ is bounded on $\ell^{p}$;

(2) $C_{\phi}$ is compact on $B^{p}(D)$ if and only if $M^{\phi}$ is compact on $\ell^{p}$.

Proof. Let

$$
f(z)=\sum_{j=1}^{\infty} \sum_{k=1}^{n_{j}} c_{j, k} k_{z_{j, k}}(z) \in B^{p}(D) \quad \text { with }\|f\|_{B^{p}(D)}^{p} \approx \sum_{j=1}^{\infty} \sum_{k=1}^{n_{j}}\left|c_{j, k}\right|^{p}
$$

and

$$
g(z)=\sum_{j=1}^{\infty} \sum_{k=1}^{n_{j}} \lambda_{j, k} k_{z_{j, k}}(z) \in B^{p^{\prime}}(D) \quad \text { with }\|g\|_{B^{p^{\prime}(D)}}^{p^{\prime}} \approx \sum_{j=1}^{\infty} \sum_{k=1}^{n_{j}}\left|c_{j, k}\right|^{p^{\prime}}
$$


Then

$$
\begin{aligned}
\left(C_{\phi}(f), g\right)_{0} & =\int_{D} V_{r}\left(C_{\phi}(f)\right)(z) \overline{\left.V_{r}(g)\right)(z)} d \lambda(z) \\
& =\int_{D} C_{\phi}(f)(z) \overline{V_{r}(g(z)} d \lambda(z) \\
& =\sum_{j=1}^{\infty} \sum_{k=1}^{n_{j}} c_{j, k} \sum_{\ell=1}^{\infty} \sum_{m=1}^{n_{\ell}} \bar{\lambda}_{\ell, m} \frac{\int_{D} C_{\phi}\left(K_{z_{j, k}}\right)(z) K_{r}\left(z, z_{\ell, m}\right) d v(z)}{K\left(z_{j, k}\right) K\left(z_{\ell, m}\right)} \\
& =\left(M^{\phi}\left\{c_{j, k}\right\},\left\{\lambda_{\ell, m}\right\}\right) .
\end{aligned}
$$

Using the duality theorems: $B^{p}(D)^{*}=B^{p^{\prime}}(D)$ under the pairing $(\cdot, \cdot)_{0}$ and the fact $\left(\ell^{p}\right)^{*}=\ell^{p^{\prime}}$, we see that $C_{\phi}$ is bounded on $B^{p}(D)$ if and only if $M^{\phi}$ is bounded on $\ell^{p}$. Similarly, we can deduce that $C_{\phi}$ is compact on $B^{p}(D)$ if and only if $M^{\phi}$ is compact on $\ell^{p}$.

Questions about if one has a nicer characterization on boundedness and compactness for $C_{\phi}$ on $B^{p}(D)$ as the case $p=1$ and $p=\infty$ are not completely answered here. We provide some partial results, and leave the problem for further study.

TheOREM 4.3. For $1<p<\infty$, we have

(1) If $C_{\phi}$ is bounded on $B^{p}(D)$, then $\sup _{z \in D}\left\|C_{\phi}\left(k_{z}\right)\right\|_{B^{p}(D)}<\infty$;

(2) If $C_{\phi}$ is compact on $B^{p}(D)$, then $\lim _{z \rightarrow \partial D}\left\|C_{\phi}\left(k_{z}\right)\right\|_{B^{p}(D)}=0$;

(3) If $C_{\phi}$ is bounded on $B^{1}(D)$, then $C_{\phi}$ is bounded on $B^{p}(D)$;

(4) If $C_{\phi}$ is compact on $B^{1}(D)$, then $C_{\phi}$ is compact on $B^{p}(D)$.

Proof. (1) and (2) easily follow from the fact that $\left\|k_{z}\right\|_{B^{p}(D)} \approx 1$ for all $z \in D$ and $k_{z}(w) \rightarrow 0$ uniformly on any compact subset of $D$.

To prove (3), recall the interpolation theorem for $B^{p}(D)$,

$$
B^{p}(D)=\left[B^{1}(D), B^{\infty}(D)=\mathcal{B}(D)\right]_{\theta}, \quad \theta=\frac{1}{p} .
$$

Thus

$$
\left\|C_{\phi}\right\|_{B^{p} \rightarrow B^{p}} \leq\left\|C_{\phi}\right\|_{B^{1}(D) \rightarrow B^{1}(D)}^{\theta}\left\|C_{\phi}\right\|_{\mathcal{B}(D) \rightarrow \mathcal{B}(D)}^{1-\theta} .
$$

Therefore, the boundedness of $C_{\phi}$ on $B^{1}(D)$ implies that $C_{\phi}$ is bounded on $B^{p}(D)$ for all $1 \leq p \leq \infty$ since $C_{\phi}$ is always bounded on $\mathcal{B}(D)$.

Next, to prove (4) suppose $C_{\phi}$ is compact on $B^{1}(D)$. Let $\left\{g_{m}\right\}_{m=1}^{\infty}$ be a sequence in $B^{p}(D)$ with $\left\|g_{m}\right\|_{B^{p}(D)}=1$ and assume $\left\{g_{m}\right\}$ converges uniformly to 0 on any compact subset in $D$. It suffices to show that

$$
\lim _{m \rightarrow \infty}\left\|C_{\phi}\left(g_{m}\right)\right\|_{B^{p}(D)}=0
$$

First, we claim the following: For any $\epsilon>0$, there is $M_{\epsilon} \geq 1$ such that

$$
\left\|C_{\phi}(f)\right\|_{B^{1}(D)}<\epsilon\|f\|_{B^{1}(D)}
$$

for all

$$
f(z)=\sum_{j=M_{\epsilon}}^{\infty} \sum_{k=1}^{n_{j}} c_{j, k} k_{z_{j, k}}(z) .
$$


Suppose the claim is not true, then there is a constant $\epsilon_{0}>0$, a sequence $\left\{M_{m}\right\}$ such that $\lim _{m \rightarrow \infty} M_{m}=\infty$ and

$$
f_{m}(z)=\sum_{j=M_{m}}^{\infty} \sum_{k=1}^{n_{j}} c_{j, k} k_{z_{j, k}}(z) \text { with }\left\|f_{m}\right\|_{B^{1}(D)}=1
$$

but $\left\|C_{\phi}\left(f_{m}\right)\right\|_{B^{1}(D)} \geq \epsilon_{0}$ for all $m$. Note that $f_{m}(z)$ converges uniformly to 0 on any compact subset of $D$ since $\sum_{j=M_{m}}^{\infty} \sum_{k=1}^{n_{j}}\left|c_{j, k}\right| \lesssim 1$ by Theorem 1.1 and $\lim _{m \rightarrow \infty} M_{m}=\infty$. From the compactness of $C_{\phi}$ on $B^{1}(D)$, we see that $\left\|C_{\phi}\left(f_{m}\right)\right\|_{B^{1}(D)} \rightarrow 0$ as $m \rightarrow \infty$. This contradicts with $\left\|C_{\phi}\left(f_{m}\right)\right\|_{B^{1}(D)} \geq \epsilon_{0}>0$. So the claim is proved.

Let $\epsilon>0$ and choose $M_{\epsilon}$ as in the claim. By Theorem 1.1, we can write

$$
g_{m}(z)=\sum_{j=1}^{\infty} \sum_{k=1}^{n_{j}} c_{j, k ; m} k_{z_{j, k}}(z) \quad \text { with }\left\|g_{m}\right\|_{B^{p}(D)}^{p} \approx \sum_{j=1}^{\infty} \sum_{k=1}^{n_{j}}\left|c_{j, k ; m}\right|^{p} .
$$

Since $\left\{g_{m}\right\}$ converges uniformly to 0 on any compact subset in $D$, for any $\epsilon>0$ there is $m_{0}$ such that

$$
\sum_{j=1}^{M_{\epsilon}} \sum_{k=1}^{n_{j}}\left|c_{j, k ; m}\right|<\epsilon
$$

for all $m \geq m_{0}$.

Consider the following subspaces of $B^{p}(D)$

$$
B^{p}(D, \epsilon)=\left\{f=\sum_{j=M_{\epsilon}}^{\infty} \sum_{k=1}^{n_{j}} c_{j, k} k_{z_{j, k}}:\left\{c_{j, k}\right\} \in \ell^{p}\right\} .
$$

Then, $C_{\phi}$ is bounded on $B^{1}(D, \epsilon)$ with $\left\|C_{\phi}\right\|_{B^{1}(D, \epsilon)} \leq \epsilon$. Applying the interpolation theorem for space $B^{p}(D, \epsilon)$, we see that

$$
\left\|\sum_{j=M_{\epsilon}}^{\infty} \sum_{k=1}^{n_{j}} c_{j, k ; m}\right\|_{B^{p}(D)} \leq C^{1 / p^{\prime}} \epsilon^{1 / p} .
$$

From (4.3) and (4.4) we can deduce that $\lim _{m \rightarrow \infty}\left\|C_{\phi}\left(g_{m}\right)\right\|_{B^{p}(D)} \rightarrow 0$ as $m \rightarrow \infty$. So, $C_{\phi}$ is compact on $B^{p}(D)$ and (4) is proved, and so is Theorem 4.3.

We now are ready to prove Theorem 1.2.

Proof of Theorem 1.2. For (1) and (2), note that

$$
M_{1}(\phi) \leq\left\|M_{\phi, 1}(\cdot)\right\|_{L^{\infty}(D)}
$$

where $M_{\phi, 1}(z)$ is defined as in (1.7). Note that the boundedness of $C_{\phi}$ on $B^{1}(D)$ implies $\left\|M_{\phi, 1}(\cdot)\right\|_{L^{\infty}(D)} \lesssim\left\|C_{\phi}\right\|<\infty$. Thus, (1) follows from (1) of Theorem 4.1. Similarly, we can deduce (2) from (2) of Theorem 4.1 .

Note that (3) and (4) follows from those of Theorem 4.3. To prove (5), let $f \in B^{p}(D)$. Then, by Theorem 1.1 we get

$$
f(z)=\sum_{j=1}^{\infty} \sum_{k=1}^{n_{j}} \lambda_{j, k} k_{z_{j, k}}(z) \quad \text { with }\|f\|_{B^{p}(D)} \approx\left(\sum_{j=1}^{\infty} \sum_{k=1}^{n_{j}}\left|\lambda_{j, k}\right|^{p}\right)^{1 / p} .
$$


Thus, we have

$$
\begin{aligned}
\left\|C_{\phi}(f)\right\|_{B^{p}(D)} & \leq \sum_{j=1}^{\infty} \sum_{k=1}^{n_{j}}\left|\lambda_{j, k}\right|\left\|C_{\phi}\left(k_{z_{j, k}}\right)\right\|_{B^{p}(D)} \\
& \leq\left(\sum_{j=1}^{\infty} \sum_{k=1}^{n_{j}}\left|\lambda_{j, k}\right|^{p}\right)^{1 / p}\left(\sum_{j=1}^{\infty} \sum_{k=1}^{n_{j}}\left\|C_{\phi}\left(k_{z_{j, k}}\right)\right\|_{B^{p}(D)}^{p^{\prime}}\right)^{1 / p^{\prime}} \\
& \lesssim\left(\sum_{j=1}^{\infty} \sum_{k=1}^{n_{j}}\left|\lambda_{j, k}\right|^{p}\right)^{1 / p}\left(\int_{D}\left\|C_{\phi}\left(k_{z}\right)\right\|_{B^{p}(D)}^{p^{\prime}} d \lambda(z)\right)^{1 / p^{\prime}} \\
& \lesssim\left(\int_{D}\left\|C_{\phi}\left(k_{z}\right)\right\|_{B^{p}(D)}^{p^{\prime}} d \lambda(z)\right)^{1 / p^{\prime}}\|f\|_{B^{p}(D)} .
\end{aligned}
$$

This implies (5) of Theorem 1.2.

To prove (6), it suffices to show that $\left\|C_{\phi}\left(f_{s}\right)\right\|_{B^{p}(D)} \rightarrow 0$ as $s \rightarrow \infty$ for every bounded sequence $\left\{f_{s}\right\}$ in $B^{p}(D)$ which converges to 0 uniformly on any compact subset of $D$. Let $\left\{f_{s}\right\}$ be a such sequence, then by Theorem 1.1

$$
f_{s}(z)=\sum_{j=1}^{\infty} \sum_{k=1}^{n_{j}} \lambda_{j, k} k_{z_{j, k ; s}}(z) \quad \text { with }\|f\|_{B^{p}(D)} \approx\left(\sum_{j=1}^{\infty} \sum_{k=1}^{n_{j}}\left|\lambda_{j, k ; s}\right|^{p}\right)^{1 / p} \approx 1 .
$$

By the assumption of (6), for any $\eta>0$ there is $\delta>0$ so that

$$
\left(\int_{D_{\delta}^{c}}\left\|C_{\phi}\left(k_{z}\right)\right\|_{B^{p}(D)}^{p^{\prime}} d v(z)\right)^{1 / p^{\prime}}<\eta
$$

where $D_{\delta}^{c}$ is the set defined in (1.8). Therefore, there is $N$ so that $z_{j, k} \in D_{\delta}^{c}$ for all $k=1, \cdots, n_{j}$ if $j \geq N$. Moreover, from the assumption that $\left\{f_{s}\right\}$ converges uniformly on compact subsets of $D$, there is $s_{0} \geq 1$ so that if $s \geq s_{0}$ then

$$
\left(\sum_{j=1}^{N} \sum_{k=1}^{n_{j}}\left|\lambda_{j, k ; s}\right|^{p}\right)^{1 / p}<\eta
$$


From (4.5) and (4.6), we have

$$
\begin{aligned}
\left\|C_{\phi}\left(f_{s}\right)\right\|_{B^{p}(D)} \leq & \sum_{j=1}^{\infty} \sum_{k=1}^{n_{j}}\left|\lambda_{j, k ; s}\right|\left\|C_{\phi}\left(k_{z_{j, k}}\right)\right\|_{B^{p}(D)} \\
\leq & \left(\sum_{j=1}^{N} \sum_{k=1}^{n_{j}}\left|\lambda_{j, k ; s}\right|^{p}\right)^{1 / p}\left(\sum_{j=1}^{N} \sum_{k=1}^{n_{j}}\left\|C_{\phi}\left(k_{z_{j, k}}\right)\right\|_{B^{p}(D)}^{p^{\prime}}\right)^{1 / p^{\prime}} \\
& +\left(\sum_{j=N}^{\infty} \sum_{k=1}^{n_{j}}\left|\lambda_{j, k ; s}\right|^{p}\right)^{1 / p}\left(\sum_{j=N}^{\infty} \sum_{k=1}^{n_{j}}\left\|C_{\phi}\left(k_{z_{j, k}}\right)\right\|_{B^{p}(D)}^{p^{\prime}}\right)^{1 / p^{\prime}} \\
\lesssim & \left(\sum_{j=1}^{N} \sum_{k=1}^{n_{j}}\left|\lambda_{j, k ; s}\right|^{p}\right)^{1 / p}\left(\int_{D}\left\|C_{\phi}\left(k_{z}\right)\right\|_{B^{p}(D)}^{p^{\prime}} d \lambda(z)\right)^{1 / p^{\prime}} \\
& +\left(\sum_{j=1}^{\infty} \sum_{k=1}^{n_{j}}\left|\lambda_{j, k ; s}\right|^{p}\right)^{1 / p}\left(\int_{D_{\delta}^{c}}\left\|C_{\phi}\left(k_{z}\right)\right\|_{B^{p}(D)}^{p^{\prime}} d \lambda(z)\right)^{1 / p^{\prime}} \\
\leq & \left(\left\|M_{\phi, p}(\cdot)\right\|_{L^{p^{\prime}}(D, d \lambda)}+1\right) \cdot \eta
\end{aligned}
$$

Since $\eta>0$ is arbitrary, this implies that $\left\|C_{\phi}\left(f_{s}\right)\right\|_{B^{p}(D)} \rightarrow 0$ as $s \rightarrow \infty$. Therefore, $C_{\phi}$ is compact on $B^{p}(D)$ proof of (6) Theorem 1.2 is complete, and so is the proof of Theorem 1.2.

\section{REFERENCES}

[1] F. Beatrous, Behavior of Holomorphic Functions near weakly Pseudoconvex Boundary Points, Indiana Univ. Math. J., 40 (1991), pp. 915-966.

[2] F. Beatrous And S.-Y. Li, On the boundedness and compactness of operators of Hankel type, J. of Functional Analysis, 111 (1993), pp. 350-379.

[3] P. S. Bourdon, J. A. Cima, and A. L. Matheson, Compact Composition Operators on BMOA, Trans. of AMS, 351 (1999), pp. 2183-2196.

[4] R. Coifman and R. Rochberg, Representation theorems for holomorphic and harmonic functions, Astérisque, 77 (1980), pp. 11-65.

[5] R. Coifman, R. Rochberg, and G. Weiss, Factorization theorem for Hardy spaces in several variables, Ann. of Math., 103 (1976), pp. 611-635.

[6] A. Cima, C. Stanton, And W. Wogen, On boundedness of composition operators on $H^{2}\left(B_{2}\right)$, Proc. Amer. Math. Soc., 99 (1984), pp. 217-222.

[7] A. Cima and W. Wogen, Unbounded composition operators on $H^{2}\left(B_{n}\right)$, Proc. Amer. Math. Soc., 99 (1987), pp. 477-483.

[8] C. C. Cowen And B. MacCluer, Composition operators on spaces of analytic functions, CRC Press, Boca Raton, New York, London, Tokyo, 1995.

[9] Z. Cuckovic And R. Zhaо, Essential Norm Estimates of Weighted Composition Operators between Bergaman Spaces on strongly Pseudoconvex Domains, Journal of London Math. Soc., 70:2 (2004), pp. 307-324..

[10] C. FEFFERMAn, The Bergman kernel and biholomorphic mappings of pseudoconvex domains, Invent. Math., 26 (1974), pp. 1-65.

[11] I. Graham, Boundary behavior of the Carathèodory and Kobayashi metric on strongly pseudoconvex domains in $\mathbb{C}^{n}$, Tran. Amer. Math. Soc., 207 (1975), pp. 219-240.

[12] S. G. Krantz, Geometric Analysis and Function Spaces, CBMS, Regional conference series in Math. 81 (1993).

[13] S. G. KRANTZ AND S.-Y. LI, A Note on Hardy spaces and functions of bounded mean oscillation on domains in $\mathbb{C}^{n}$, Mich. Math. J., 41 (1994), pp. 51-72.

[14] S. G. KRantz And S.-Y. Li, Duality theorems on Hardy and Bergman spaces on convex domains of finite type in $\mathbb{C}^{n}$, Ann of Fourier Institute, 45 (1995), pp. 1305-1327. 
[15] S. G. Krantz And S.-Y. LI, On the decomposition theorems for Hardy spaces in domains in $\mathbb{C}^{n}$ and application, J. of Fourier Analysis and Applications, 2 (1995), pp. 65-107.

[16] S. G. Krantz, S.-Y. Li, And R. Rochberg, Analysis of some function spaces associated to Hankel operators, Ill. Jour. of Math., 41 (1997), pp. 398-411.

[17] F. JAFARI, Composition operators in Bergman spaces on bounded symmetric domains, Cont. Math., 137 (1992), pp. 277-290.

[18] S.-Y. LI, Composition Operators and Isometries on Holomorphic Function Spaces over Domains in $\mathbb{C}^{n}$, The Proceedings of the International Conference on Complex Geometry and Related Fields, AMS/IP Studies in Advanced Math., IP press, 39 (2007), pp. 161-174.

[19] S.-Y. LI, Trace Ideal criteria for composition operators on Bergman spaces, Amer. J. of Math, 117 (1995), pp. 1299-1324.

[20] S.-Y. LI, Some function theoretical results on domains in $\mathbb{C}^{n}$, Proceeding of ICCM 2001, Editors: C.-S. Lin, L. Yang and S.-T. Yau, International press, 2004.

[21] S.-Y. Li AND S. Long, Compact composition operators on $B M O A\left(B_{n}\right)$, Science in China, 52 (2009), pp. 2679-2687.

[22] S.-Y. Li And S. Long, Compact composition operators on Block space $\mathcal{B}\left(B_{n}\right)$, Houston Math. Journal, to appear.

[23] S.-Y. Li AND W. Luo, On characterization of Besov space and application. Part I, J. of Math. Analysis and Applications, 310 (2005), pp. 477-491.

[24] S.-Y. Li And W. Luo, Analysis on Besov spaces II: Embedding and Duality Theorems, J. of Math. Analysis and Applications, 333:2 (2007), pp. 1189-1202.

[25] S.-Y. Li And B. Russo, On the compactness of composition operators on Hardy spaces in strongly pseudoconvex domains in $\mathbf{C}^{n}$, Proceeding of A.M.S., 123 (1995), pp. 161-172.

[26] D. H. Luecking, Representation and duality in the weighted spaces of analytic function, Indiana Univ. Math. J., 34 (1985), pp. 319-336.

[27] D. H. Luecking And K. Zhu, Composition operators belonging to the Schatten ideals, Amer. J. Math., 114 (1992), pp. 1127-1145.

[28] B. MacCluer, Composition operators on $H^{p}\left(B_{n}\right)$, Mich. Math. J., 32 (1985), pp. 237-248.

[29] B. MacCluer, Spectra of compact composition operators on $H^{p}\left(B_{N}\right)$, Analysis, 4 (1984), pp. $87-103$.

[30] B. MacCluer and J. H. Shapiro, Angular derivatives and compact composition operators on Hardy and Bergman spaces, Can. J. Math., 38 (1986), pp. 878-906.

[31] B. Russo, Holomorphic Composition Operators in Several Complex Variables, Contemporary Mathematics, 213 (1998), pp. 191-212.

[32] J. H. Shapiro, The essential norm of a composition operator, Ann. Math., 125 (1987), pp. 375404.

[33] J. H. Shapiro, Composition Operators ad Classical Function Theory, Springer-Verlag, ew York, 1993.

[34] J. H. Shapiro And P. D. TAYlor, Compact, nuclear and Hilbert-Schmidt composition operators on $H^{2}$, Indiana U. Math. J., 23 (1973), pp. 471-496.

[35] W. Sмiтh, Compactness of composition operators on BMOA, Proc. Amer. Math. Soc., 127:9 (1999), pp. 2715-2725.

[36] H. Wulan, Compactness of composition operators on BMOA and VMOA, Sci. China Ser. A, 50:7 (2007), pp. 997-1004.

[37] Z.-H. Zhou AND J. H. SHI, Compactness of composition operators on the Bloch space in classical bounded symmetric domains, Michigan Math. J., 50:2 (2002), pp. 381-405.

[38] R. Zhao, Composition Operators from Bloch Type Spaces to Hardy Spaces, Jour. of Math. Anal. And Appl., 233 (1999), pp. 749-766.

[39] K. ZHu, Operator theory in function spaces, Math. Surverys and Monographs, 138, Second edition, New York: Amer. Math. Soc, 2007. 
